\title{
Disruption of the Ang II type 1 receptor promotes longevity in mice
}

\author{
Ariela Benigni,, ${ }^{1}$ Daniela Corna, ${ }^{1}$ Carla Zoja, ${ }^{1}$ Aurelio Sonzogni, ${ }^{2}$ Roberto Latini, ${ }^{3}$ Monica Salio, ${ }^{3}$ \\ Sara Conti, ${ }^{1}$ Daniela Rottoli, ${ }^{1}$ Lorena Longaretti,, ${ }^{1}$ Paola Cassis, ${ }^{1}$ Marina Morigi, ${ }^{1}$ \\ Thomas M. Coffman, ${ }^{4}$ and Giuseppe Remuzzi ${ }^{1,5}$

\begin{abstract}
1"Mario Negri" Institute for Pharmacological Research, Bergamo, Italy. 2Department of Pathology, Azienda Ospedaliera Ospedali Riuniti di Bergamo, Bergamo, Italy. 3“Mario Negri” Institute for Pharmacological Research, Milan, Italy. ${ }^{4}$ Department of Medicine, Duke University Medical Center and Durham Veterans Affairs Medical Center, Durham, North Carolina, USA.
\end{abstract} \\ ${ }^{5}$ Unit of Nephrology and Dialysis, Azienda Ospedaliera Ospedali Riuniti di Bergamo, Bergamo, Italy.
}

\begin{abstract}
The renin-angiotensin system plays a role in the etiology of hypertension and the pathophysiology of cardiac and renal diseases in humans. Ang II is the central product of this system and is involved in regulating immune responses, inflammation, cell growth, and proliferation by acting through Ang II type 1 receptors (AT 1 and $\mathrm{AT}_{2}$ ). Here, we show that targeted disruption of the Agtr1a gene that encodes $\mathrm{AT}_{1 \mathrm{~A}}$ results in marked prolongation of life span in mice. Agtr1 $a^{-/-}$mice developed less cardiac and vascular injury, and multiple organs from these mice displayed less oxidative damage than wild-type mice. The longevity phenotype was associated with an increased number of mitochondria and upregulation of the prosurvival genes nicotinamide phosphoribosyltransferase (Nampt) and sirtuin 3 (Sirt3) in the kidney. In cultured tubular epithelial cells, Ang II downregulated Sirt 3 mRNA, and this effect was inhibited by an $\mathrm{AT}_{1}$ antagonist. These results demonstrate that disruption of $\mathrm{AT}_{1}$ promotes longevity in mice, possibly through the attenuation of oxidative stress and overexpression of prosurvival genes, and suggests that the Ang $\mathrm{II} / \mathrm{AT}_{1}$ pathway may be targeted to influence life span in mammals.
\end{abstract}

\section{Introduction}

High blood pressure is highly prevalent in humans and is associated with complications, including heart disease, kidney disease, and stroke, which together represent the major (and neglected) health problems for high- and middle-income countries that are now impending on low-income countries (1). The renin-angiotensin system (RAS) has critical functions in regulation of blood pressure and cardiovascular physiology (2). Its role in the pathogenesis of a number of disease states has been well documented, and components of the RAS are important pharmacological targets in hypertension and cardiovascular disease. Responsiveness to Ang II is conferred by the expression of 2 classes of pharmacologically distinct rhodopsin-like $\mathrm{G}$ protein-associated receptors, the Ang II type 1 and 2 receptors $\left(\mathrm{AT}_{1}\right.$ and $\left.\mathrm{AT}_{2}\right)(3)$. The effect of Ang II to increase blood pressure and to promote various pathologies is mediated by $\mathrm{AT}_{1}$, which is expressed in various organ systems, including the heart, blood vessels, kidney, adrenal glands, and cardiovascular control centers in the brain (4). Targeted disruption of the Agtrla gene encoding $\mathrm{AT}_{1 \mathrm{~A}}$ - the major mouse $\mathrm{AT}_{1}$ isoform and mouse homolog to the single human AGTR1 gene (5) - causes a reduction in blood pressure levels. Here, we investigated whether inactivation of Agtrla gene in mice also confers protection from cardiovascular morbidity and mortality. The present study documented that inhibition of $\mathrm{AT}_{1}$ signaling promotes longevity.

\section{Results}

Inactivation of $A T_{1 A}$ prolongs the life span of mice. A prospective observational study was performed in 20 homozygous mice deficient

Conflict of interest: The authors have declared that no conflict of interest exists.

Nonstandard abbreviations used: $\mathrm{AT}_{1}$, Ang II type 1 receptor; BUN, blood urea nitrogen; Nampt, nicotinamide phosphoribosyltransferase; PTEC, proximal tubular epithelial cell; RAS, renin-angiotensin system; Sirt3, sirtuin 3.

Citation for this article: J. Clin. Invest. 119:524-530 (2009). doi:10.1172/JCI36703. for $\mathrm{AT}_{1 \mathrm{~A}}$ - the major mouse $\mathrm{AT}_{1}$ isoform and the closest murine homolog to the single human $\mathrm{AT}_{1}(5)$ - and 10 wild-type controls. The animals lacking $\mathrm{AT}_{1 \mathrm{~A}}$ substantially outlived their wild-type littermates (Figure 1). At 29 months, when all wild-type animals died, $17 \mathrm{AT}_{1 \mathrm{~A}}$-deficient mice (85\%) were still alive. These remaining mice lived for an additional 7 months. Comparison of survival curves showed a highly significant difference between groups $(P<0.0001)$. The life span of the $\mathrm{AT}_{1 \mathrm{~A}}$-deficient mice was approximately $26 \%$ longer than controls.

Caloric restriction is associated with increased longevity (6-8). To assess whether caloric intake was effected by the $\mathrm{AT}_{1 \mathrm{~A}}$ mutation, daily food intake was carefully monitored at 19 to 21 months of age. Food intake was virtually identical between $\mathrm{Agtr} \mathrm{a}^{-/-}$and wild-type mice (Table 1). Small body size is also associated with extended life span in diet-restricted mice (9). Body weights were slightly but not significantly reduced in Agtr $1 a^{-/}$compared with wild-type littermates until month 18 when they became indistinguishable (Figure 2A). Agtr1 $a^{-/}$mice had normal physical activity as reflected by their ability to perform on a rotarod (Table 1 ). Adenocarcinoma of the lung was observed in $10 \%-15 \%$ of animals in both aging cohorts, an incidence of lung tumor not correlated with genotype and lower than previously reported $(\sim 30 \%)$ in C57BL/6 × 129 mice (10).

$A T_{1 A}$ knockout mice develop less cardiac and vascular injury. Animals were sacrificed when death appeared imminent, and the heart, aorta, pancreas, kidney, liver, spleen, and brain were collected to assess pathophysiological consequences of $\mathrm{AT}_{1 \mathrm{~A}}$ deficiency on organ structure. Morphological examination of the various tissues revealed major differences between Agtr1 $a^{-/}$(26 to 30 months old) and wild-type animals (26 to 29 months old) in the heart, aorta, and pancreas. The heart weight/body weight ratio and left ventricular mass were comparable between Agtr1 $a^{-/-}$and wild-type mice (Figure 3, A and B). No difference was found in the number 


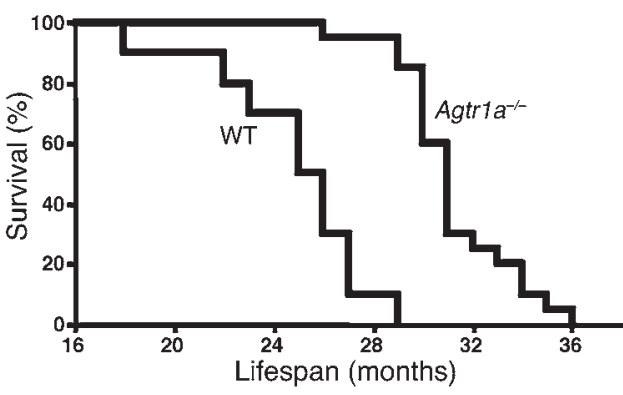

Figure 1

Knocking-out $\mathrm{AT}_{1 \mathrm{~A}}$ prolongs life span in the mouse. A Kaplan-Meier analysis of survival in male Agtr1 $\mathrm{a}^{-/-}$animals and wild-type littermates is shown (log-rank test, 20.32; $P<0.0001$ versus wild-type mice). The average life span of ggtr1a $^{-/-}$animals and wild-type littermates was $31.20 \pm 2.31$ and $24.81 \pm 3.10$ months, respectively.

of cardiomyocytes in transverse sections of left ventricle. By contrast, myocyte cross-sectional area, an index of myocyte size, was significantly lower in Agtr1a $a^{-/}$than in wild-type mice (Figure 3C). Furthermore, cardiac fibrosis was significantly reduced in $\mathrm{AT}_{1 \mathrm{~A}^{-}}$ deficient animals compared with wild-type controls. For example, morphological analysis of serial heart slices showed that deposition of collagen in interstitial spaces was reduced in the Agtrla $a^{-/-}$ animals (Figure 3D). Agtr1 $a^{-/}$mice did not develop lesions throughout the aorta (Figure 4A). By contrast, $50 \%$ of wild-type mice showed a 2-fold increase in atherosclerotic lesions compared with Agtrla $a^{-1-}$ animals. Atherosclerotic lesions were characterized by fragmentation of lamina elastica of the vessel wall (Figure 4B) and intraparietal accumulation of foaming cells destroying layers of elastic tunica media (Figure 4C).

While pancreata from Agtr1 $1 a^{-/-}$mice appeared normal morphologically, wild-type littermates manifested lymphoid aggregates that encompassed a substantial proportion of the islets. However, the aggregates rarely penetrated the islets and were not associated with necrosis. To determine whether insulitis in wild-type mice resulted in a loss of $\beta$ cells, staining for insulin was performed in pancreatic tissue. The extent of staining for insulin was virtually identical in Agtr1 $a^{-/}$and wild-type mice, thereby indicating a comparable number of insulin-secreting cells. There was no difference in fasting glucose levels between Agtr1 $a^{-/}$and wild-type mice (Figure 2B).

Kidney histology showed mild tubular pathology, effecting less than $25 \%$ of tubuli in few animals of both strains. Changes in glomeruli, including mesangial/intracapillary hypercellularity and mesangiolysis, were not different in Agtrla $a^{-/}$and wild-type mice. Analysis of the liver revealed mild hydropic degeneration of hepatocytes in Agtrlat-/ mice. At variance, in wild-type animals marked hydropic degeneration of hepatocytes, macro- and microvesicular steatosis and mild exudation were observed. Mild vascular congestion of the red pulp of the spleen was observed in both strains. Mild edema of the white substance of the brain was present in both Agtr1 $a^{-/-}$and wild-type mice at a comparable extent.

Renal function is normal in $A T_{1 A}$ knockout mice. Urinary protein excretion levels were within the normal range in $A g t r 1 a^{-/-}$and wildtype mice ( 24 months old, $4.81 \pm 4.19$ versus $5.56 \pm 2.42 \mathrm{mg} /$ day, respectively). Twenty-four-hour urinary volumes were relatively constant in both groups but were higher in Agtr1a ${ }^{-/-}$mice (1-1.6 $\mathrm{ml} /$ day) compared with the wild-type group (0.6-1 ml/day), consistent with an impaired urinary concentrating mechanism described previously in these animals (11). Renal function did not change during the study and was comparable between groups (blood urea nitrogen [BUN] at 24 months old, Agtr1 $a^{-/-}, 21.5 \pm 7.17$ versus wildtype, $19.1 \pm 6.31 \mathrm{mg} / \mathrm{dl}$ ).

Lack of $A T_{1 A}$ reduces oxidative damage and prevents age-induced mitochondrial loss. Accumulation of oxidative damage inflicted by reactive oxygen species is the major cause of aging in invertebrates and mammalians (12). An excess formation of peroxynitrite, the reaction product of nitric oxide and superoxide anion $\left(\mathrm{O}_{2}^{-}\right)$, has been taken to reflect increased lipid peroxidation and oxidation of structural proteins (13). The expression of nitrotyrosine, a marker of peroxynitrite production, was increased in cardiomyocytes, aortas, and kidneys obtained from aged animals (26 to 29 months old) compared with 2-month-old mice (Figure 5, A and B). By contrast, nitrotyrosine staining was reduced in cardiomyocytes, aortas, and kidneys from Agtr1 $a^{-1-}$ mice (26 to 30 months old) with respect to aged wildtype mice; however, these values were not significantly different from those of 2-month-old mice (Figure 5, A and B), suggesting that absence of $\mathrm{AT}_{1 \mathrm{~A}}$ protects tissues from oxidative damage.

Mitochondria, the main cellular source of reactive oxygen species, are particularly sensitive to oxidant damage. Kidney proximal tubular cells possess large numbers of mitochondria and are highly dependent on mitochondrial energy production for proper function (14). In wild-type mice, the numerical density of mitochondria in proximal tubule cells was significantly reduced $(P<0.01)$ in aged (26 to 29 months old) compared with 2-month-old animals (Figure 6 , A and $\mathrm{B}$ ). A lack of $\mathrm{AT}_{1 \mathrm{~A}}$ protected mice from this loss of mitochondria as animals aged $(P<0.01$; Figure 6 , A and $B)$. Abnormalities in mitochondria density were not accompanied with changes in mean mitochondria volume (Figure 6B).

Longevity of $A T_{1 A}$ knockout mice is associated with increased renal expression of survival genes. Studies have shown that in the context of nutrient restriction, mitochondria dictate cell survival through the upregulation of nicotinamide phosphoribosyltransferase (Nampt), which by boosting mitochondrial $\mathrm{NAD}^{+}$provides the cosubstrate for SIRT3, a member of the sirtuin family linked to life span extension $(15,16)$. We therefore evaluated whether Nampt and Sirt3, which are highly expressed in mitochondria, were differentially regulated in long-term surviving mice lacking $\mathrm{AT}_{1 \mathrm{~A}}$. Quantitative real-time PCR showed that transcript levels of both Nampt and Sirt3 were significantly increased in kidneys from Agtr1 $a^{-/-}$mice (26 to 30 months old) with respect to wild-type animals (26 to 29 months old) (Figure 7). Since overexpression of SIRT1 modulates mitochondrial biogenesis (17), transcript levels of Sirt1 were also evaluated. No change in Sirt1 mRNA expression was detected in kidneys from Agtrla $a^{-/}$mice (26 to 30 months old) with respect to wild-type animals (26 to 29 months old) (Figure 7).

Ang II downregulates Nampt and Sirt3 expression via $A T_{1 A}$ in cultured mouse proximal tubular epithelial cells. To determine whether Ang II

\section{Table 1}

Food intake and latency to fall from rotarod in WT and $\mathrm{Agtr1a}^{-1}$ mice were determined using mice at 19 to 21 months of age

\begin{tabular}{lcr} 
& WT mice & \multicolumn{1}{c}{ Agtr1a $^{-/}$mice } \\
Food intake $(\mathrm{g})$ & $3.8 \pm 0.4(n=9)$ & $3.9 \pm 0.8(n=20)$ \\
Rotarod (s) & $124.0 \pm 10.0(n=6)$ & $129.0 \pm 10.0(n=6)$ \\
\hline
\end{tabular}

Data are mean \pm SD. 

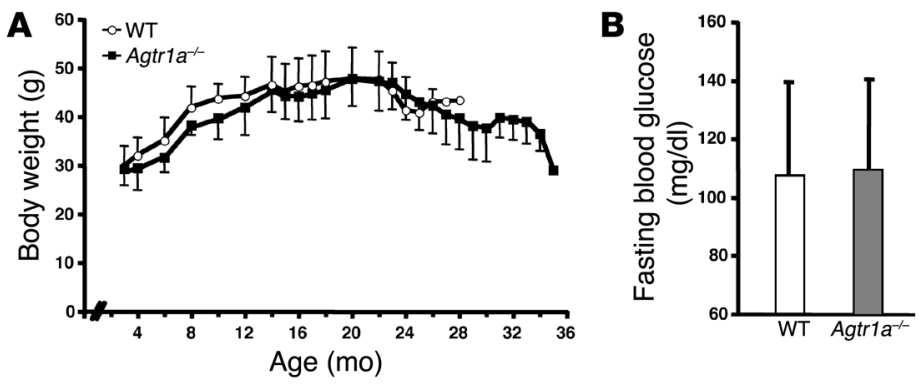

\section{Figure 2}

Body weight and blood glucose levels in $\mathrm{Agtr1}^{-/-}$and wild-type mice. (A) Body weight in Agtr1 $^{-/-}$animals and wild-type littermates. No significant difference in weight gain was observed. (B) Fasting blood glucose levels were comparable in Agtr1 $^{-/-}$ animals $(n=9)$ and wild-type littermates $(n=6)$ at month 24 . Data are mean \pm SD. directly effects the expression of Nampt and Sirt3 via $\mathrm{AT}_{1}$, in vitro experiments were performed in mouse proximal tubular epithelial cells (PTECs) exposed to Ang II in the presence or absence of candesartan. Nampt and Sirt3 mRNA expression, evaluated by realtime PCR, was downregulated by Ang II challenge. Preincubation of mouse PTECs with candesartan prevented the reduction of Nampt and Sirt3 transcript expression in response to Ang II (Figure 8A). To further evaluate whether Ang II-induced downregulation of Nampt could impact Sirt3 expression, we assessed Sirt3 expression in PTECs transfected with specific Nampt siRNA. Twenty-five nanomoles of Nampt siRNA effectively reduced the expression of Nampt mRNA by $67 \%$ (Figure 8B). Silencing the Nampt gene prevented the decrease of Sirt 3 mRNA induced by Ang II, thereby indicating a causative role of Nampt in Sirt3 expression.

\section{Discussion}

In the present study, we have found that targeted disruption of $\mathrm{AT}_{1 \mathrm{~A}}$ translated into marked prolongation of the life span of mice. We used the F1 progeny of the (C57BL/6 × 129/SvEv)Agtr1a-/strain, generated by intercrossing 2 inbred lines, which is ideal for the aging studies because animals lack structural or developmental abnormalities. Furthermore, since each F1 mouse has the same genotype across the entire genome, they are genetically pure; each F1 animal is genetically identical to the next. This would obviously change in subsequent crosses, and, therefore, we used only F1 (C57BL/ $6 \times 129 / \mathrm{SvEv})$ mice differing at the Agtr1a gene locus ( + or - ). A theoretical limitation of the use of animals with a mixedstrain background is the effect of strain-specific genes linked to the targeted allele. In fact, the null allele of Agtr1 $a^{-/-}$strain could contain a small amount of tightly linked genomic DNA from 129 not present in the wild-type B6 allele. However, because the life spans of wild-type 129 and C57BL/6 are very similar, it seems highly unlikely that a strain-specific gene linked to the 129 Agtrla locus could explain the robust survival differences seen in our studies. Moreover, some have argued against using inbred mouse lines for aging studies, since many of these lines harbor recessive mutations predisposing to tumors or other disorders that independently effect survival. In this regard, F1 mice may offer advantages over the parental inbred lines for this sort of experiment.

Animals lacking $\mathrm{AT}_{1 \mathrm{~A}}$ developed fewer aortic atherosclerotic lesions and less cardiac damage, as reflected by the reduction in myocyte size and fibrosis, with lower deposition of interstitial collagen with respect to wild-type mice. This is in line with recent studies investigating the role of Ang II in end-organ damage, showing that a knockin mouse model with gain-of-function mutation of $\mathrm{AT}_{1}$ developed progressive cardiac fibrosis with increased expression of collagen (18). These results point to a direct action of Ang II through $\mathrm{AT}_{1 \mathrm{~A}}$ in regulating extracellular matrix production by cardiac fibroblasts (19).
Oxidative stress is a principal contributor to aging. In the fly and the mouse, gene mutations that enhance resistance to environmental stresses extend life span (12). Ang II increases vascular $\mathrm{O}_{2}{ }^{-}$ production and induces endothelial nitric oxide synthase enzyme uncoupling, which may further enhance oxidative stress in vascular tissue, thereby decreasing nitric oxide availability. Nitric oxide, by interacting with $\mathrm{O}_{2}{ }^{-}$, forms peroxynitrite, a cytotoxic anion that inhibits mitochondrial electron transport (20). The observation in the present study of reduced production of peroxynitrite in hearts, aortas, and kidneys from Agtr1a/- compared with wild-type mice would imply that Ang II effected organ senescence through induction of oxidative stress via $\mathrm{AT}_{1}$.

Oxidative damage to mitochondria could account for some of the age-related changes in their function. Tissues from aging animals have a reduced number of mitochondria (14) that show functional changes, including increased superoxide and $\mathrm{H}_{2} \mathrm{O}_{2}$ generation and decreased energy production (21). An important finding of the present study was that prolonged survival of $A g t r 1 a^{-/-}$mice

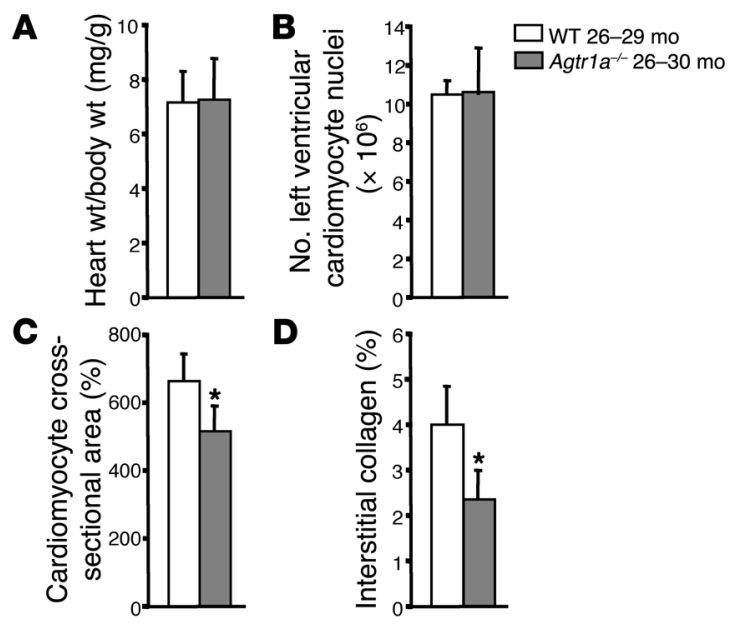

Figure 3

$\mathrm{AT}_{1 \mathrm{~A}}$ deficiency protects heart from hypertrophy and fibrosis. Paraffin-embedded hearts (Agtr1 $a^{-/-}$mice, $n=7,26$ to 30 months old; wildtype mice, $n=4,26$ to 29 months old) were sectioned at $5 \mu \mathrm{m}$ for hematoxylin-eosin for morphometric studies and at $10 \mu \mathrm{m}$ for collagen content stained with picrosirius solution. (A) Neither the ratio of heart weight/body weight, (B) nor the estimated number of left ventricular cardiomyocytes were effected by the absence of $A T_{1 A}$. (C) The crosssectional area of cardiomyocytes, an index of hypertrophy, and (D) interstitial collagen, a marker of cardiac fibrosis, were significantly lower in Agtr1 $^{-l-}$ mice. Data are mean \pm SD. ${ }^{\star} P<0.01$ versus wildtype mice by Student's $t$ test for impaired data. 

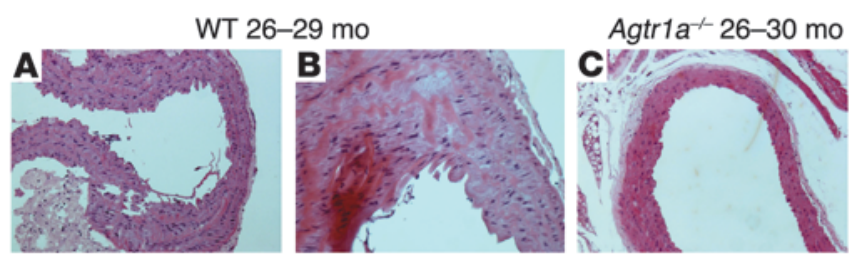

\section{Figure 4}

Lack of $\mathrm{AT}_{1 \mathrm{~A}}$ protects mice from aorta damage. (A and $\left.\mathbf{B}\right)$ Representative images of aorta sections from wild-type mice (26 to 29 months old), showing fragmentation of lamina elastica along all the perimeter of the vessel wall (A) and intraparietal accumulation of foamy cells destroying layers of elastic tunica media (B). (C) Aorta section from Agtr1 $a^{-/-}$mice (26 to 30 months old) showing regular elastic lamina. Original magnification, $\times 20$.

was associated with preservation of the number of mitochondria in the renal PTECs. These results are consistent with previous descriptions of a beneficial effect of angiotensin-converting enzyme inhibition to preserve mitochondria in aging mice (22).

Mitochondrial SIRT3 and SIRT4, members of histone deacetylase family that use $\mathrm{NAD}^{+}$as cosubstrate, appear to play a role in the longevity network that maintains mitochondrial vitality (23). In the context of nutrient restriction, the expression of the Nampt gene, the mammalian equivalent of the yeast longevity gene PNC1, increases. Enhanced Nampt expression leads to the accumulation of its biosynthetic product $\mathrm{NAD}^{+}$in mitochondria, which in turn promotes cell survival via activation of mitochondrial SIRT3 and SIRT4 (16). Here, we wondered whether high levels of Ang II measured in Agtr1a-- mice (24) could induce Nampt gene, which in turn may have prevented age-induced mitochondria death, as it occurs as a consequence of caloric restriction (16). We found that both Nampt and Sirt3 were upregulated in the kidneys of long-term surviving mice lacking $\mathrm{AT}_{1 \mathrm{~A}}$ with respect to wild-type littermates. Our data would suggest that Nampt and Sirt3 may play a role in promoting mitochondria vitality in Agtr1 $1 a^{-/}$mice, contributing to prolong survival. Whether increased sirtuin expression is the effect rather than the cause of increased mitochondrial maintenance is worth investigating. The finding that candesartan prevented Ang IIinduced Nampt and Sirt3 mRNA reduction in cultured tubular epi- thelial cells can be taken to suggest a possible biochemical link between Ang II and survival genes, which conceivably operates via $\mathrm{AT}_{1}$. Furthermore, experiments showing that Nampt gene silencing by siRNA limited the reduction of Sirt $3 \mathrm{mRNA}$ induced by Ang II would indicate a causative role of Nampt in modulating Sirt3 gene transcription in response to Ang II.

Data are available that indicate that life span extension in mice is associated with reduced insulin receptor signaling cascade, which upregulates the expression of sirtuin proteins $(15,25)$. There is the possibility that $\mathrm{AT}_{1 \mathrm{~A}}$ deficiency reduces oxidation of the components of insulin receptor signaling pathway, which may enhance SIRT3 expression (26). The extension of life span described here is comparable to that of mice lacking the insulin growth factor-1 (IGF-1) receptor (27) or the p66 isoform of the proto-oncogene Shc, the major transduction molecule of IGF-1 receptor (28). Of note, prolonged survival induced by resveratrol (29) - a small molecule found in red wine that activates Sirt 1 and mimics the antiaging effect of caloric restriction - is associated with increased mitochondrial number (29) and is dependent on the upregulation of Sir2 (30). Moreover, resveratrol downregulates $\mathrm{AT}_{1}$ through Sirt1 activation in cultured vascular smooth muscle cells and mouse aorta, implying that inhibition of $\mathrm{AT}_{1}$ contributes to resveratrol-induced longevity (31). The finding here that Sirt1 mRNA transcript levels were comparable in the kidneys from Agtrla- and wild-type mice would suggest that the longevity pattern described here is independent from Sirt1 pathway.

Sirtuins can be therefore regarded as a potential therapeutic target for diseases of aging and to extend life. This is consistent with findings that Sir2 homologs do actually prolong life span in worms and flies, mimicking the life-prolonging effects of caloric restriction (30). Activators of Sirt1 ameliorate insulin resistance and enhance mitochondrial capacity in experimental diabetes (32). However, manipulating the sirtuin pathway in humans is not imminently feasible. In contrast, $\mathrm{AT}_{1}$ antagonists have been proven to be safe and well tolerated for chronic use and are used as a key component of the modern therapy for hypertension and cardiac failure (33). Data are also available that suggest that these molecules share renal and cardiac protective properties $(33,34)$. Specifically, long-term inhibition of the RAS by either angiotensin-converting enzyme inhibitors or Ang II receptor antagonist protected rats from the deleterious process of aging on the car-

\section{Figure 5}

Absence of $A T_{1 A}$ limits oxidative damage. (A) Staining scores of nitrotyrosine, a marker of peroxynitrite, in heart, aorta, and kidney were increased in aged wild-type mice (26 to 29 months old) with respect to young wild-type mice (2 months old). Deficiency of $\mathrm{AT}_{1 \mathrm{~A}}$ resulted in less oxidative stress. ${ }^{\dagger} P<0.05$ versus wild-type 2 -month-old mice; ${ }^{*} P<0.05$ versus wild-type 26 - to 29-month-old mice. Data are mean \pm SD. (B) Representative images of kidney sections from wild-type 2-month-old, wildtype 26- to 29-month-old, and Agtr1a - $^{-1}$ 26- to 30-month-old animals. Peroxynitrite was localized with a specific anti-nitrotyrosine antibody in the tubulointerstitial area. Peroxynitrite staining was increased in wild-type 26- to 29-month-old animals with respect to wild-type 2-month-old animals. Very weak staining was found in Agtr1a ${ }^{-/}$mice, which was comparable to wild-type 2-month-old animals. Original magnification, $\times 400$.

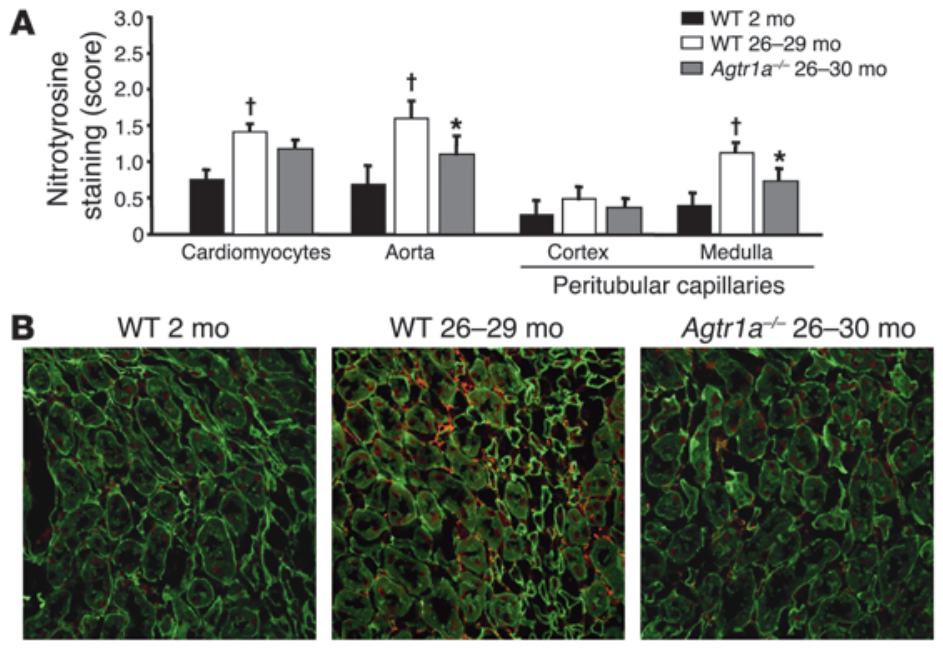



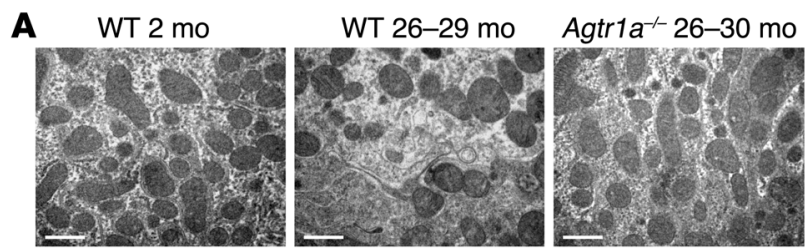

B
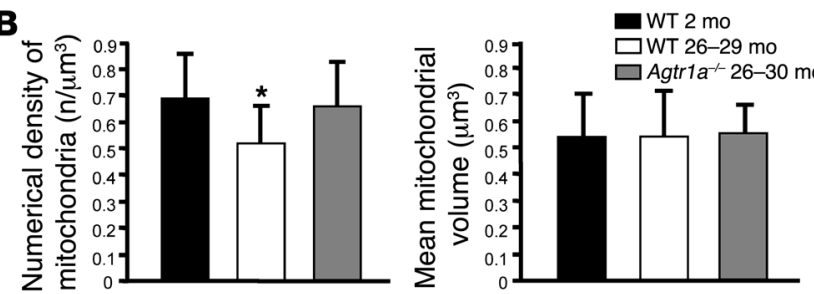

\section{Figure 6}

$\mathrm{AT}_{1 \mathrm{~A}}$ deficiency prevents loss of mitochondria. (A) Representative transmission electron micrographs of the ultrastructure of mouse proximal tubular cells obtained from resin-embedded kidney sections from young wild-type mice (2 months old), aged wild-type mice (26 to 29 months old), and Agtr1 $^{-/-}$animals (26 to 30 months old). Scale bar: $1 \mu \mathrm{m}$. (B) The number of mitochondria per volume in proximal tubular cells in aged wild-type animals was decreased with respect to wild-type 2-month-old and Agtr1 $\mathrm{a}^{-/-}$mice. Agtr1a $\mathrm{a}^{-/}$animals showed the same numerical density as wild-type 2 -month-old animals. ${ }^{*} P<0.01$ versus wild-type 2-month-old and Agtr1 ${ }^{-/-}$animals by ANOVA corrected with Bonferroni coefficient. Mean mitochondria volume evaluated on the same transmission electron micrographs through morphometrical analysis did not differ among groups. Data are mean \pm SD.

diovascular system and induced a substantial extension of animal life span (34).

In summary, we have provided evidence that $\mathrm{AT}_{1 \mathrm{~A}}$ deficiency increases life span in mice. Longevity was likely the consequence of reduced mitochondrial damage due to the attenuation of oxidative stress and the upregulation of Nampt and Sirt3 survival genes. Our study offers a rationale for exploring the possibility that $\mathrm{AT}_{1}$ antagonists prolong life in humans.

\section{Methods}

Mice. Male F1 $(\mathrm{C} 57 \mathrm{BL} / 6 \times 129 / \mathrm{SvEv})$ mice lacking $\mathrm{AT}_{1 \mathrm{~A}}$ for Ang II were obtained and bred in the animal facility of the Durham Veterans Affairs Medical Center under the NIH guidelines (5) and then maintained in the animal facility of the "Mario Negri" Institute for Pharmacological Research in conformity with the institutional guidelines that are in compliance with national (Decreto Legislativo n.116, Gazzetta Ufficiale suppl 40, 18 febbraio 1992, Circolare n.8, Gazzetta Ufficiale 14 luglio 1994) and international laws and policies (EEC Council Directive 86/609, OJL358-1, December 1987; Guide for the care and use of laboratory animals. NIH publication no. 85-23. Revised 1996). All animal studies were approved by the Institutional Animal Care and Use Committees of "Mario Negri" Institute for Pharmacological Research. After generating the targeted disruption of the Agtrla gene locus (35), this mutation was back-crossed for multiple generations $(>12)$ onto several inbred genetic backgrounds, including C57BL/6 and 129/SvEv. However, renal structural abnormalities were found in $\mathrm{AT}_{1 \mathrm{~A}}$-deficient mice on these 2 backgrounds (36), making them unsuitable for physiological studies. By contrast, kidney abnormalities were not seen in F1(C57BL/6 × 129/SvEv)Agtr1 $1 \mathrm{a}^{-/-}$mice. The lack of structural or developmental abnormalities along with their genetic homogeneity render the F1(C57BL/6 × 129/SvEv)Agtr1a-/- mice ideal for the aging studies carried out here. Animals were housed in a constant temperature room, with a 12-hour dark/12-hour light cycle, and allowed free access to standard diet (4RF21 GLP certificate, Mucedola srl; $12.0 \%$ water, $18.5 \%$ protein, $3.0 \%$ fat, $6.0 \%$ fiber, $7.0 \%$ ash; metabolizable energy, $2,668 \mathrm{kCal} / \mathrm{kg}$ ) and water. Heart, aorta, pancreas, kidney, liver, spleen, lung, and brain specimens were collected for histological analysis from $7 \mathrm{Agtr}^{-\mathrm{a}^{-/-}}$mice ( 26 to 30 months old) and 4 wild-type mice ( 26 to 29 months old) that were killed when death appeared imminent. Organs were collected in $10 \%$ formalin, except for the pancreas (collected in Bouin's solution) and the kidney (collected in Duboscq-Brazil), embedded in paraffin, and sectioned at $3 \mu \mathrm{m}$ for hematoxylin-eosin staining. For morphometrical analysis of mitochondria and oxidative stress evaluation, additional 2-month-old wild-type mice $(n=4)$ were considered.

Biochemical measurements. Blood glucose levels were measured with a reflectance meter (A. Menarini Diagnostics) at month 24, after overnight fasting. Renal function was assessed as BUN in heparinized blood by the Reflotron test (Roche Diagnostics). BUN levels of more than $30 \mathrm{mg} / \mathrm{dl}$ were considered abnormal. Twenty-four-hour urine samples were collected using clean metabolic cages, and proteinuria was determined by the Coomassie protein assay reagent (Sigma-Aldrich), using bovine serum albumin as standard.

Immunofluorescence for insulin. Sections $(3 \mu \mathrm{m})$ from OCT frozen pancreatic tissue were incubated with rabbit polyclonal anti-insulin antibody (1:100; Santa Cruz Biotechnology Inc.) followed by goat anti-rabbit FITCconjugated antibody (1:50; Jackson ImmunoResearch Laboratories Inc.).

Heart morphometry. Tissue was formalin-fixed, paraffin-embedded, and sectioned at $5 \mu \mathrm{m}$ for hematoxylin-eosin and at $10 \mu \mathrm{m}$ for collagen staining $(0.1 \%$ Sirius red in picric acid). Cardiomyocyte cross-sectional area was measured by manually tracing the cell contour on images acquired on the Image Analyzer IBAS 2.0 (Kontron/Zeiss image analysis system) at $\times 400$ magnification from at least 50 cardiomyocytes in each section (37). The total number and volume of cardiomyocytes were determined by quantitative morphometric analysis (38). Collagen content was quantified in digitally acquired images and expressed as fractional area of the entire section. The nature of Sirius red-stained collagen deposit was confirmed by examination under a microscope equipped with polarized light that renders collagen fibers birefringent. Interstitial collagen was quantified as described (37).

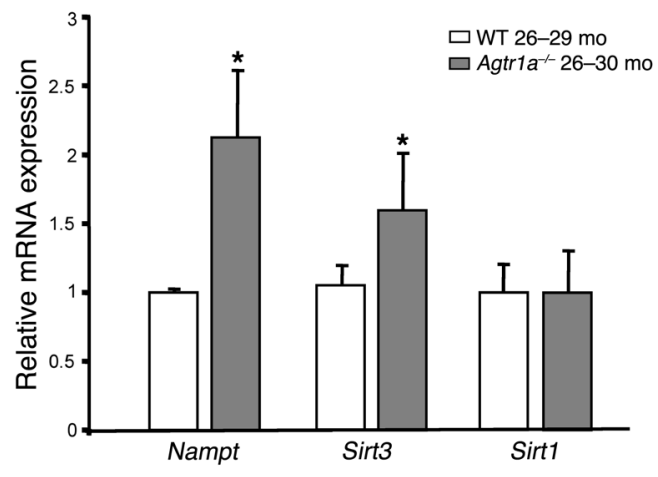

\section{Figure 7}

Lack of $A T_{1 A}$ is associated with increased levels of Nampt and Sirt3 in the kidney. By real-time PCR, whole kidney expression of both Nampt and Sirt3 was increased in Agtr1 a $^{-1-}$ (26 to 30 months old) with respect to wild-type (26 to 29 months old) mice. Renal expression of Sirt1 mRNA was comparable in the 2 strains. Data are mean \pm SD of 4 animals. ${ }^{*} P<0.05$ versus wild-type by ANOVA corrected with Bonferroni coefficient. 


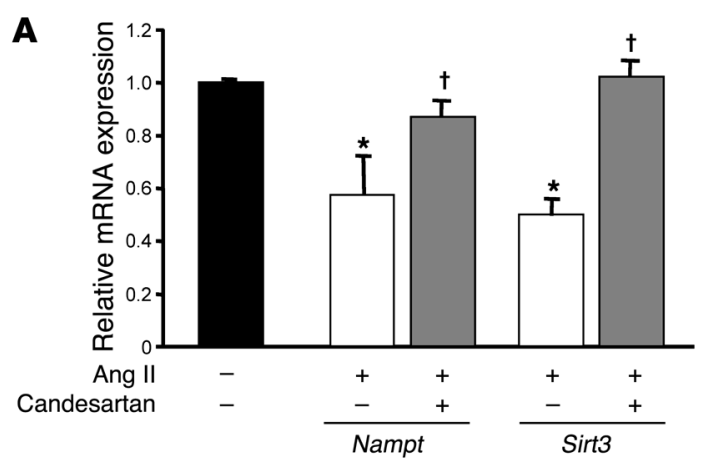

B
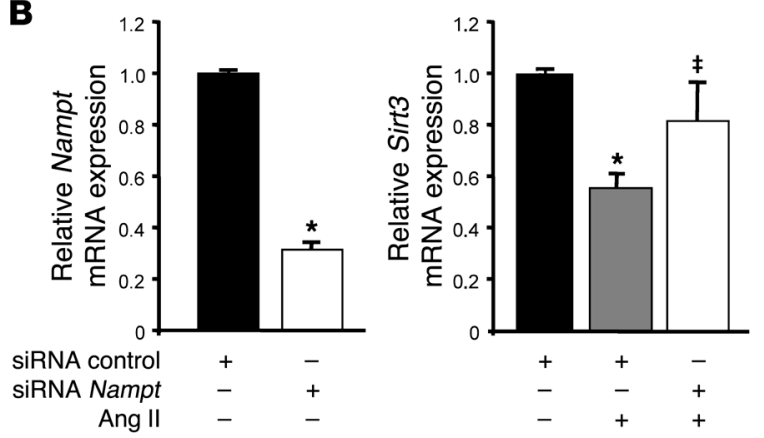

Immunohistochemistry for nitrotyrosine staining. Paraffin-embedded aorta and heart sections $(3 \mu \mathrm{m})$ were treated with $0.3 \%$ Triton X-100/PBS and incubated overnight with rabbit polyclonal anti-nitrotyrosine IgG (1:500; Upstate Biotechnology) and further with biotinylated goat anti-rabbit (1:200) and Vectastain $\mathrm{ABC}$ kit and DAB reagents (Vector Laboratories). Frozen kidney sections $(3 \mu \mathrm{m}$ ) were heated for 20 minutes in $10 \mathrm{mM}$ citrate buffer ( $\mathrm{pH}$ 6.0) for antigen retrieval. After incubation with rabbit polyclonal anti-nitrotyrosine $\operatorname{IgG}(1: 500)$, the sections were incubated with goat anti-rabbit Cy3-coniugated antibody (1:100; Jackson ImmunoResearch Laboratories Inc.) and counterstained with lectin FITC-conjugated antibody (1:400; Vector Laboratories). Ten to twenty fields (magnification, $\times 200$ ) of aorta or renal tubuli for each section were analyzed, and the scoring ( 0 , absent; 1 , faint; 2 , moderate; 3 , intense) of nitrotyrosine staining was calculated as a weighed mean.

Estimation of mitochondrial numerical density and mean volume. Glutaraldehyde-fixed fragments of cortical kidney tissue were washed in cacodylate buffer, postfixed in $1 \% \mathrm{OsO}_{4}$, dehydrated, and embedded in Epon resin. Ultrathin sections were stained with uranyl acetate for examination, using a Philips Morgagni transmission electron microscope (Philips). Numerical density of mitochondria $\left(N_{V}, n / \mu \mathrm{m}^{3}\right)$ was estimated by morphometrical analysis (39), using an orthogonal grid digitally superimposed over digitized electron microscope pictures of proximal tubules at $\times 7,100$. Mitochondrial profile area density $\left(N_{A}\right)$ was estimated by the ratio between the number of mitochondria and the proximal tubular area in the image calculated on the basis of grid points. Mitochondrial volume density $\left(V_{V}\right)$ was determined by the ratio of grid points falling over mitochondria divided by the total number of points of the grid contained in proximal tubule section. $N_{V}$ was then estimated for each animal using the formula $N_{V}=(1 / \beta)$ $\left(N_{A}^{3 / 2} / V_{V}^{1 / 2}\right)$, in which $\beta$ is the shape coefficient for ellipsoidal mitochondria, calculated from the ratio of the harmonic mean of major and minor axis of mitochondria sections measured on digital images. The mean mitochondrial volume was calculated for each animal as the ratio of mitochondrial volume density $V_{V}$ and numerical density $N_{V}$.

Cell culture and incubation. SV40-transformed murine PTEC lines (provided by Eric G. Neilson, Vanderbilt University, Nashville, Tennessee,

\section{Figure 8}

Effect of Ang II on prosurvival genes in mouse cultured PTECs. (A) Ang II downregulates Nampt and Sirt3 mRNA via AT ${ }_{1}$. After 24-hour incubation with serum-free DMEM, PTECs were incubated with candesartan $(0.1 \mu \mathrm{M})$ or medium for 1 hour and then exposed to Ang II $(1 \mu \mathrm{M})$ or medium (control) for 3 hours. The black column represents unstimulated cells maintained in medium (control) for 4 hours. Nampt and Sirt3 mRNA levels were evaluated by real-time PCR. Data are mean \pm SD of 3 experiments. ${ }^{*} P<0.01$ versus control, ${ }^{t} P<0.01$ versus Ang II by ANOVA corrected with Bonferroni coefficient. (B) Nampt modulates Sirt3 gene transcription in response to Ang II. After 6 hour transfection with Nampt siRNA, cells were maintained in medium for 45 hours and then incubated 3 hours with Ang II. Data are mean \pm SD of 3 experiments. ${ }^{*} P<0.01$ versus control, $¥ P<0.01$ versus siRNA control plus Ang II by Student's $t$ test for unpaired data (left panel) or ANOVA corrected with Bonferroni coefficient (right panel).
USA) were grown in DMEM (Sigma-Aldrich) plus 10\% FCS (Invitrogen) supplemented with L-glutamine (2 mM; Invitrogen), penicillin (100 U/ml; Invitrogen), and streptomycin $(100 \mu \mathrm{g} / \mathrm{ml}$; Invitrogen $)$. Cells were maintained in serum-free DMEM for 24 hours before and during the experiments (16). To investigate the effect of Ang II on Nampt, Sirt1, and Sirt3 gene expression, PTECs were exposed for 3 hours to medium (control) or $1 \mu \mathrm{M}$ Ang II (Calbiochem) in the presence or absence of the $\mathrm{AT}_{1}$ antagonist candesartan $(0.1 \mu \mathrm{M}$; AstraZeneca). Cells were incubated with candesartan 1 hour before and during the incubation with Ang II.

$R N A$ interference. The Nampt-specific siRNA sequence (5'-UAAUUGGAUACCAGGACUGaa- $3^{\prime}$ ) was designed according to the complete sequence of mouse Nampt gene (GenBank accession number 10946947). In addition, a 19-bp scrambled siRNA, a sequence with no significant homology to any known gene sequences from mouse, rat, or human, was used as negative control for RNA interference. siNampt (Silencer Select Pre-designed siRNAs, catalog no. s81814) and negative control siRNA (catalog no. 4611) were synthesized by Ambion.

For silencing of Nampt, PTECs grown on 6 -well plates $\left(2 \times 10^{5}\right.$ cells/well $)$ were transfected with Nampt siRNA ( $25 \mathrm{nM}$ ) or negative control siRNA using Lipofectamine 2000 (Invitrogen) according to the manufacturer's instruction. After 6 hours of treatment with the siRNA reaction mixture, RNA/lipid complexes were removed and cells were incubated in DMEM for a further 45 hours. To investigate the effect of Ang II on Nampt and Sirt3 gene expression after treatment with siRNA, PTECs were exposed for 3 hours to medium (control) or $1 \mu \mathrm{M}$ Ang II, then collected and used for RNA extraction.

Real-time PCR of Nampt, Sirt1, and Sirt3. Total RNA was extracted from kidneys and PTECs using TRIzOL reagent according to the manufacturer's instruction (Invitrogen). Purified RNA $(2 \mu \mathrm{g})$ was reverse transcribed. To amplify cDNA, TaqMan Universal PCR Master Mix (Applied Biosystems) was used according to the manufacturer's instruction and inventoried TaqMan assay Mn01293560_m1 was used for Nampt, Mn01168521_m1 was used for Sirt1, and Mn01275638_m1 was used for Sirt3, and a mouse $\beta$-actin endogenous control (VIC/MGB probe) was also used. The PCR was performed on the 7300 Real-Time PCR System (Applied Biosystems). We 
used the $\Delta \Delta$ Ct technique to calculate cDNA content in each sample using the cDNA expression in mouse wild-type or control PTECs as calibrator.

Statistics. Data are expressed as mean \pm SD. The time occurrence of death (months) was assessed by Kaplan-Meier cumulative survival functions. Time-to-event (death) was calculated in months. Survival distribution between the 2 groups of mice was compared by log-rank test. ANOVA with Bonferroni correction or the 1-tailed Student's $t$ test for unpaired data were used as appropriate. $P$ values of less than 0.05 were considered to be statistically significant.

\section{Acknowledgments}

We thank Monica Locatelli and Cristina Zanchi for biochemical assessments, Mauro Abbate for renal histological analysis, Samantha Solini for nitrotyrosine experiments, Simona Buelli for in vitro experiments, and Andrea Remuzzi for his precious advice on morphometry of mitochondria. Sara Conti is a recipient of a fellowship from Fondazione Aiuti per la Ricerca sulle Malattie Rare (ARMR) in memory of Lidia D'Arcangelo, Bergamo, Italy, and Paola Cassis is a recipient of a fellowship from Associazione Ricerca Trapianti (ART), Milan, Italy.

Received for publication July 7, 2008, and accepted in revised form December 17, 2008.

Address correspondence to: Ariela Benigni, "Mario Negri” Institute for Pharmacological Research, Via Gavazzeni 11, 24125 Bergamo, Italy. Phone: 39-035-319-888; Fax: 39-035-319-331; E-mail: abenigni@marionegri.it.
1. Chobanian, A.V., et al. 2003. Seventh report of the Joint National Committee On Prevention, Detection, Evaluation, and Treatment Of High Blood Pressure. Hypertension. 42:1206-1252.

2. Coffman, T.M., and Crowley, S.D. 2008. Kidney in hypertension: Guyton redux. Hypertension. 51:811-816.

3. Timmermans, P.B., et al. 1993. Angiotensin II receptors and angiotensin II receptor antagonists. Pharmacol. Rev. 45:205-251.

4. Kim, S., and Iwao, H. 2000. Molecular and cellular mechanisms of angiotensin II-mediated cardiovascular and renal diseases. Pharmacol. Rev. 52:11-34.

5. Oliverio, M.I., et al. 1998. Reduced growth, abnormal kidney structure, and type 2 (AT2) angiotensin receptor-mediated blood pressure regulation in mice lacking both AT1A and AT1B receptors for angiotensin II. Proc. Natl. Acad. Sci. U. S. A. 95:15496-15501.

6. Weindruch, R., Walford, R.L., Fligiel, S., and Guthrie, D. 1986. The retardation of aging in mice by dietary restriction: longevity, cancer, immunity and lifetime energy intake. J. Nutr. 116:641-654.

7. Roth, G.S., Ingram, D.K., and Lane, M.A. 1995. Slowing ageing by caloric restriction. Nat. Med. 1:414-415.

8. Piper, M.D., and Bartke, A. 2008. Diet and aging. Cell Metab. 8:99-104.

9. Brown-Borg, H.M., Borg, K.E., Meliska, C.J., and Bartke, A. 1996. Dwarf mice and the ageing process. Nature. 384:33.

10. Haines, D.C., Chattopadhyay, S., and Ward, J.M. 2001. Pathology of aging B6;129 mice. Toxicol. Pathol. 29:653-661.

11. Oliverio, M.I., et al. 2000. Abnormal water metabolism in mice lacking the type $1 \mathrm{~A}$ receptor for ANG II. Am. J. Physiol. Renal Physiol. 278:F75-F82.

12. Finkel, T., and Holbrook, N.J. 2000. Oxidants, oxidative stress and the biology of ageing. Nature. 408:239-247.

13. Viappiani, S., and Schulz, R. 2006. Detection of specific nitrotyrosine-modified proteins as a marker of oxidative stress in cardiovascular disease. Am.J. Physiol. Heart Circ. Physiol. 290:H2167-H2168.

14. De Cavanagh, E.M., et al. 2003. Enalapril and losartan attenuate mitochondrial dysfunction in aged rats. FASEB J. 17:1096-1098.

15. Christensen, K., Johnson, T.E., and Vaupel, J.W. 2006. The quest for genetic determinants of human longevity: challenges and insights. Nat. Rev. Genet. 7:436-448.

16. Yang, H., et al. 2007. Nutrient-sensitive mitochondrial NAD+ levels dictate cell survival. Cell. 130:1095-1107.

17. Lagouge, M., et al. 2006. Resveratrol improves mitochondrial function and protects against metabolic disease by activating SIRT1 and PGC-1alpha. Cell. 127:1109-1122.

18. Billet, S., et al. 2007. Gain-of-function mutant of angiotensin II receptor, type $1 \mathrm{~A}$, causes hypertension and cardiovascular fibrosis in mice. J. Clin. Invest. 117:1914-1925.

19. Matsusaka, T., Katori, H., Inagami, T., Fogo, A., and Ichikawa, I. 1999. Communication between myocytes and fibroblasts in cardiac remodeling in angiotensin chimeric mice. J. Clin. Invest. 103:1451-1458.

20. Radi, R., Beckman, J.S., Bush, K.M., and Freeman, B.A. 1991. Peroxynitrite-induced membrane lipid peroxidation: the cytotoxic potential of superoxide and nitric oxide. Arch. Biochem. Biophys. 288:481-487.

21. Sohal, R.S., Ku, H.H., Agarwal, S., Forster, M.J., and Lal, H. 1994. Oxidative damage, mitochondrial oxidant generation and antioxidant defenses during aging and in response to food restriction in the mouse. Mech. Ageing Dev. 74:121-133.

22. Ferder, L., Inserra, F., Romano, L., Ercole, L., and Pszenny, V. 1993. Effects of angiotensin-converting enzyme inhibition on mitochondrial number in the aging mouse. Am. J. Physiol. 265:C15-C18.

23. Dali-Youcef, N., Lagouge, M., Froelich, S., Koehl, C., Schoonjans, K., and Auwerx, J. 2007. Sirtuins: The 'Magnificent Seven', function, metabolism and longevity. Ann. Med. 39:335-345.

24. Li, X.C., Navar, L.G., Shao, Y., and Zhuo, J.L. 2007. Genetic deletion of AT1A receptors attenuates intracellular accumulation of ANG II in the kidney of AT1A receptor-deficient mice. Am. J. Physiol. Renal Physiol. 293:F586-F593.

25. Sauve, A.A., Wolberger, C., Schramm, V.L., and Boeke, J.D. 2006. The biochemistry of sirtuins. Annu. Rev. Biochem. 75:435-465.
26. Droge, W., and Schipper, H.M. 2007. Oxidative stress and aberrant signaling in aging and cognitive decline. Aging Cell. 6:361-370.

27. Holzenberger, M., et al. 2003. IGF-1 receptor regulates lifespan and resistance to oxidative stress in mice. Nature. 421:182-187.

28. Migliaccio, E., et al. 1999. The P66shc adaptor protein controls oxidative stress response and life span in mammals. Nature. 402:309-313.

29. Baur, J.A., et al. 2006. Resveratrol improves health and survival of mice on a high-calorie diet. Nature. 444:337-342.

30. Howitz, K.T., et al. 2003. Small molecule activators of sirtuins extend Saccharomyces cerevisiae lifespan. Nature. 425:191-196.

31. Miyazaki, R., et al. 2008. SIRT1, a longevity gene, downregulates angiotensin II type 1 receptor expression in vascular smooth muscle cells. Arterioscler. Thromb. Vasc. Biol. 28:1263-1269.

32. Milne, J.C., et al. 2007. Small molecule activators of SIRT1 as therapeutics for the treatment of type 2 diabetes. Nature. 450:712-716.

33. Remuzzi, G., Schieppati, A., and Ruggenenti, P. 2002. Clinical practice. Nephropathy in patients with type 2 diabetes. N. Engl. J. Med. 346:1145-1151.

34. Basso, N., et al. 2007. Protective effect of long-term angiotensin II inhibition. Am. J. Physiol. Heart Circ. Physiol. 293:H1351-H1358.

35. Ito, M., et al. 1995. Regulation of blood pressure by the type $1 \mathrm{~A}$ angiotensin II receptor gene. Proc. Natl. Acad. Sci. U. S. A. 92:3521-3525.

36. Le, T.H., et al. 2004. Modifier locus on mouse chromosome 3 for renal vascular pathology in AT1A receptor-deficiency. Hypertension. 43:445-451.

37. Masson, S., et al. 1998. Remodelling of cardiac extracellular matrix during beta-adrenergic stimulation: upregulation of SPARC in the myocardium of adult rats. J. Mol. Cell. Cardiol. 30:1505-1514.

38. Anversa, P., Beghi, C., Kikkawa, Y., and Olivetti, G. 1985. Myocardial response to infarction in the rat. Morphometric measurement of infarct size and myocyte cellular hypertrophy. Am. J. Pathol. 118:484-492.

39. Weibel, E. 1979. Practical methods for biological morphometry. In Stereological methods. Academic Press. London. 40-116. 